\title{
The evolution of the spatially resolved metal abundance in galaxy clusters up to $z=1.4^{\star}$ (Research Note)
}

\author{
S. Ettori ${ }^{1,2}$, A. Baldi ${ }^{3}$, I. Balestra ${ }^{4}$, F. Gastaldello ${ }^{5,6}$, S. Molendi $^{5}$, and P. Tozzi ${ }^{7}$ \\ 1 INAF, Osservatorio Astronomico di Bologna, via Ranzani 1, 40127 Bologna, Italy \\ e-mail: stefano.ettori@oabo.inaf.it \\ 2 INFN, Sezione di Bologna, viale Berti Pichat 6/2, 40127 Bologna, Italy \\ 3 Physics and Astronomy Dept., Michigan State University, East Lansing, MI 48824, USA \\ ${ }^{4}$ INAF, Osservatorio Astronomico di Trieste, via G.B. Tiepolo 11, 34131 Trieste, Italy \\ 5 INAF-IASF, via Bassini 15, 20133 Milan, Italy \\ ${ }^{6}$ Department of Physics and Astronomy, University of California at Irvine, 4129 Frederick Reines Hall, Irvine, CA 92697-4575, \\ USA \\ 7 INAF, Osservatorio Astrofisico di Arcetri, Largo Enrico Fermi 5, 50125 Firenze, Italy
}

Received 5 December 2014 / Accepted 1 April 2015

\section{ABSTRACT}

\begin{abstract}
Context. We present the combined analysis of the metal content of 83 objects in the redshift range 0.09-1.39, and spatially resolved in the three bins $(0-0.15,0.15-0.4,>0.4) R_{500}$, as obtained with similar analysis using XMM-Newton data in our previous two papers. Aims. By combining these two large data sets, we investigate the relations between abundance, temperature, radial position and redshift holding in the intracluster medium.

Methods. We fit functional forms to the combination of the different physical quantities of interest, i.e., intracluster medium (ICM) metal abundance, radius, and redshift. We use the pseudo-entropy ratio to separate the cool core (CC) cluster population, where the central gas density tends to be relatively higher, cooler and more metal rich, from the non-cool core systems.

Results. The average, redshift-independent, metal abundance measured in the three radial bins decreases moving outwards, with a mean metallicity in the core that is even three (two) times higher than the value of 0.16 times the solar abundance in Anders \& Grevesse (1989, Geochim. Cosmochim. Acta, 53, 197) estimated at $r>0.4 R_{500}$ in CC (NCC) objects. We find that the values of the emission-weighted metallicity are well fitted by the relation $Z(z)=Z_{0}(1+z)^{-\gamma}$ at the given radius. A significant scatter, intrinsic to the observed distribution and of the order of $0.05-0.15$, is observed below $0.4 R_{500}$. The nominal best-fit value of $\gamma$ is significantly different from zero $(>3 \sigma)$ in the inner cluster regions $(\gamma=1.6 \pm 0.2)$ and in CC clusters only. These results are also confirmed with a bootstrap analysis, which provides a still significant negative evolution in the core of CC systems $(P>99.9$ per cent, when counting the number of random repetitions, which yields $\gamma>0)$. No redshift evolution is observed when regions above the core $\left(r>0.15 R_{500}\right)$ are considered. A reasonable good fit of both the radial and redshift dependence is provided from the functional form $Z(r, z)=Z_{0}(1+$ $\left.\left(r / 0.15 R_{500}\right)^{2}\right)^{-\beta}(1+z)^{-\gamma}$, with $\left(Z_{0}, \beta, \gamma\right)=(0.83 \pm 0.13,0.55 \pm 0.07,1.7 \pm 0.6)$ in CC clusters and $(0.39 \pm 0.04,0.37 \pm 0.15,0.5 \pm 0.5)$ for NCC systems.

Conclusions. Our results represent the most extensive study of the spatially resolved metal distribution in the cluster plasma as function of redshift. Our study defines the limits that numerical and analytic models describing the metal enrichment in the ICM have to meet.
\end{abstract}

Key words. galaxies: clusters: intracluster medium - X-rays: galaxies: clusters

\section{Introduction}

The hot, thin X-ray emitting plasma in galaxy clusters (i.e. the intracluster medium, ICM) is enriched with metals ejected form supernovae $(\mathrm{SNe})$ explosions through subsequent episodes of star formation and subsequent diffusion through several mechanisms, e.g., ram pressure stripping, galactic winds, outflows from active Galactic nuclei, galaxy-galaxy interactions (e.g. Renzini 1997; Schindler \& Diaferio 2008).

X-ray observations provide direct measurements of the metal abundance in the ICM, as well as their radial distribution and

\footnotetext{
* The full data set is only available at the CDS via anonymous ftp to cdsarc.u-strasbg.fr (130.79.128.5) or via http://cdsarc.u-strasbg.fr/viz-bin/qcat?J/A+A/578/A46
}

variation as a function of time. These measures represent the "footprint" of cosmic star formation history and are crucial to tracing the effect of SN feedback on the ICM at different cosmic epochs (e.g. Ettori 2005; Calura et al. 2007; Cora et al. 2008; Fabjan et al. 2010).

Several studies have addressed the radial distributions of metals in the ICM at low redshift (e.g. Finoguenov et al. 2000; De Grandi \& Molendi 2001; Irwin \& Bregman 2001; Tamura et al. 2004; Baldi et al. 2007; Leccardi \& Molendi 2008; Snowden et al. 2008). A few others have constrained the evolution of the metal abundance at $z \gtrsim 0.3$ (e.g. Balestra et al. 2007; Maughan et al. 2008; Anderson et al. 2009; a statistical analysis of the combination of these different data sets is presented in Andreon 2012). In Baldi et al. (2012a), we have presented 
the XMM-Newton analysis of 39 galaxy clusters at $0.4<z<1.4$, covering a temperature range of $2 \lesssim k T \lesssim 12.8 \mathrm{keV}$. We were able to resolve their abundance in three radial bins.

In this work, we combine this data set with that presented in Leccardi \& Molendi (2008), which includes 44 objects at $z<0.31$, with gas temperatures between $2.9 \mathrm{keV}$ and $11.3 \mathrm{keV}$. The analysis performed on the XMM-Newton data in the two samples is identical and can be statistically combined to probe the ICM abundance as a function of radial position and redshift.

We adopt a cosmological model with $H_{0}=70 \mathrm{~km} \mathrm{~s}^{-1} \mathrm{Mpc}^{-1}$, $\Omega_{\mathrm{m}}=0.3$, and $\Omega_{\Lambda}=0.7$ throughout. Solar abundance values published in Anders \& Grevesse (1989) are adopted for reference. Confidence intervals are quoted at $1 \sigma$ unless otherwise stated.

\section{Data analysis}

Details on the X-ray data reduction and analysis are presented in Leccardi \& Molendi (2008) and Baldi et al. (2012a), which include a discussion on the systematic effects that could affect our estimates of the ICM metallicity. Most of these measurements, which are for high temperature $(k T>2 \mathrm{keV})$ systems at medium-high redshifts $(z>0.1)$, are mainly based on the strength of the iron $\mathrm{K}$ line (at the rest-frame energies of $6.6-7.0 \mathrm{keV})^{1}$. We briefly mention here that we performed a simultaneous spectral fit on spectra accumulated from the two MOS detectors over the energy range $0.7-8.0 \mathrm{keV}$, using a Cash statistics applied to the source plus background counts, with a modelled background emission. The free parameters of the thermal model apec in XSPEC (Arnaud 1996) are temperature, abundance, and normalization. Local absorption is fixed to the Galactic neutral hydrogen column density, as obtained from radio data (Kalberla et al. 2005), and the redshift to the value is measured from optical spectroscopy.

Because of the better statistics in lower redshift clusters, the X-ray spectral analysis of most objects in Leccardi \& Molendi (2008) was performed using a finer radial binning with respect to Baldi et al. (2012a). Thus, we had to degrade the spatial resolution of Leccardi \& Molendi (2008) abundance profiles to match its resolution with Baldi et al. (2012a) and obtain uniform abundance profiles in three radial bins: $0-0.15 R_{500}, 0.15-0.4 R_{500}$, and $>0.4 R_{500}$, where $R_{500}$ is estimated through a scaling relation (e.g. Vikhlinin et al. 2006; see Baldi et al. 2012 for details) by using a global gas temperature that does not include the core emission $\left(<0.15 R_{500}\right)$. We first carried out the spatial degradation by interpolating the abundance profile (and its relative error) over the radial points of the observed surface brightness profile $S(r)$ of each cluster. Then, we used the surface brightness profile as a weighting factor for the interpolated abundance profile, together with the errors on the abundance profile itself, so that - in each resulting bin - regions with higher surface brightness and smaller uncertainties in the measure of the abundance would have a larger weight in the computation of the average abundance. This scheme reproduces an emissionweighted profile of the metal abundance. Hydrodynamical simulations have shown that an emission-weighted metallicity matches the original value in simulations at better than $5 \%$ (e.g. Rasia et al. 2008). Following this approach, the average

\footnotetext{
1 The full data set analysed here is available at http://pico.bo. astro.it/ settori/abun/xmm. dat and at the CDS.
}

abundance $\langle Z\rangle$, and the relative error $\sigma_{\langle Z\rangle}$, in the $R_{\min }-R_{\max }$ bin was computed as:

$$
\langle Z\rangle=\frac{\sum_{i=1}^{n} w_{i} Z_{i}}{\sum_{i=1}^{n} w_{i}}, \quad \sigma_{\langle Z\rangle}=\left(\frac{\sum_{i=1}^{n} S_{i}}{\sum_{i=1}^{n} w_{i}}\right)^{0.5},
$$

where $Z_{i}$ and $\sigma_{Z_{i}}$ are the abundance profile and the error on the abundance profile, respectively, $w_{i}=S_{i} / \sigma_{Z_{i}}^{2}$ is the weighting factor, and the sum is done over the $n$ radial bins falling in the radial range $R_{\min }-R_{\max }$ in the abundance profile of each cluster, as measured in Leccardi \& Molendi (2008).

\section{Results on the spatially resolved abundance evolution}

This work presents our final effort to use archived data to constrain the evolution of the spatially resolved ICM abundance. Our analysis separates the central regions, where cool cores (if any) appear, and the rest of the cluster atmosphere. We require that the spectrum from which the metallicity is measured contains at least 300 net counts. We consider a total of 221 data points corresponding to the spatially resolved spectroscopic measurements of the metal abundance distribution in 83 galaxy clusters in the redshift range 0.092-1.393. By estimating the pseudo-entropy ratio $\sigma=\left(T_{0} / T_{1}\right) \times\left(E M_{0} / E M_{1}\right)^{-1 / 3}$, where $T_{0}$ and $T_{1}$ are the temperatures measured in the $r<0.15 R_{500}$ region and in the $0.15-0.4 R_{500}$ annulus, respectively, and $E M_{0}$ and $E M_{1}$ are the corresponding emission measures, we define as non-cool core (NCC) clusters 54 (out of 83) objects that have $\sigma \geq 0.6$ (see e.g. Leccardi et al. 2010; Baldi et al. 2012b). The data points are plotted as function as radius and redshift, separating between CC and NCC systems, in Fig. 1. All the results are presented in Table 1.

In the $r<0.15 R_{500}$ radial bin, we have 70 data points over the entire redshift range. The distribution of the metal abundance correlates significantly with the redshift (Spearman's rank $\rho_{z}=-0.25$, corresponding to a significance of $2.1 \sigma$ versus the null-hypothesis of no correlation; see Table 2), with the temperature $\left(\rho_{T}=-0.3,2.5 \sigma\right)$ and with the pseudo-entropy ratio $\left(\rho_{\sigma}=-0.4,3.3 \sigma\right)$. These correlations point towards a significant effect induced from the cooling activities taking place in these regions, where the ICM at high density radiates more efficiently, lowering its global temperature and increasing its metal budget (see e.g. Leccardi \& Molendi 2008). Fitting the data points in this bin with a power law in the form $Z \propto(1+z)^{-\gamma}$, we obtain $\gamma=1.60 \pm 0.22$, which differs from zero at high significance $(>6 \sigma)$. When a bootstrap analysis is done, i.e. the fit is repeated $10^{5}$ times after a random sampling with replacement and the median, we consider the 1st and 3rd quartiles to estimate location and dispersion of the distribution of the best-fitting parameters. We also find that the central values are recovered, but the relative errors are increased by about a factor of 2 , mostly because a more proper sampling of the intrinsic scatter is performed with the bootstrap analysis. The negative evolution $(\gamma>0)$ is still detected in $>99.9$ per cent of the replica. When we consider CC and NCC systems separately, the normalization $Z_{0}$ is different by a factor of 2.2 in favour of CC systems, where a negative evolution is still detected at very high significance. Instead, no evolution is measured $(\gamma=0.5 \pm 0.4)$ in NCC objects, although the case for negative evolution is still observed in about $80 \%$ of the bootstrapped repetitions. 
S. Ettori et al.: The evolution of the metal abundance up to $z=1.4(R N)$
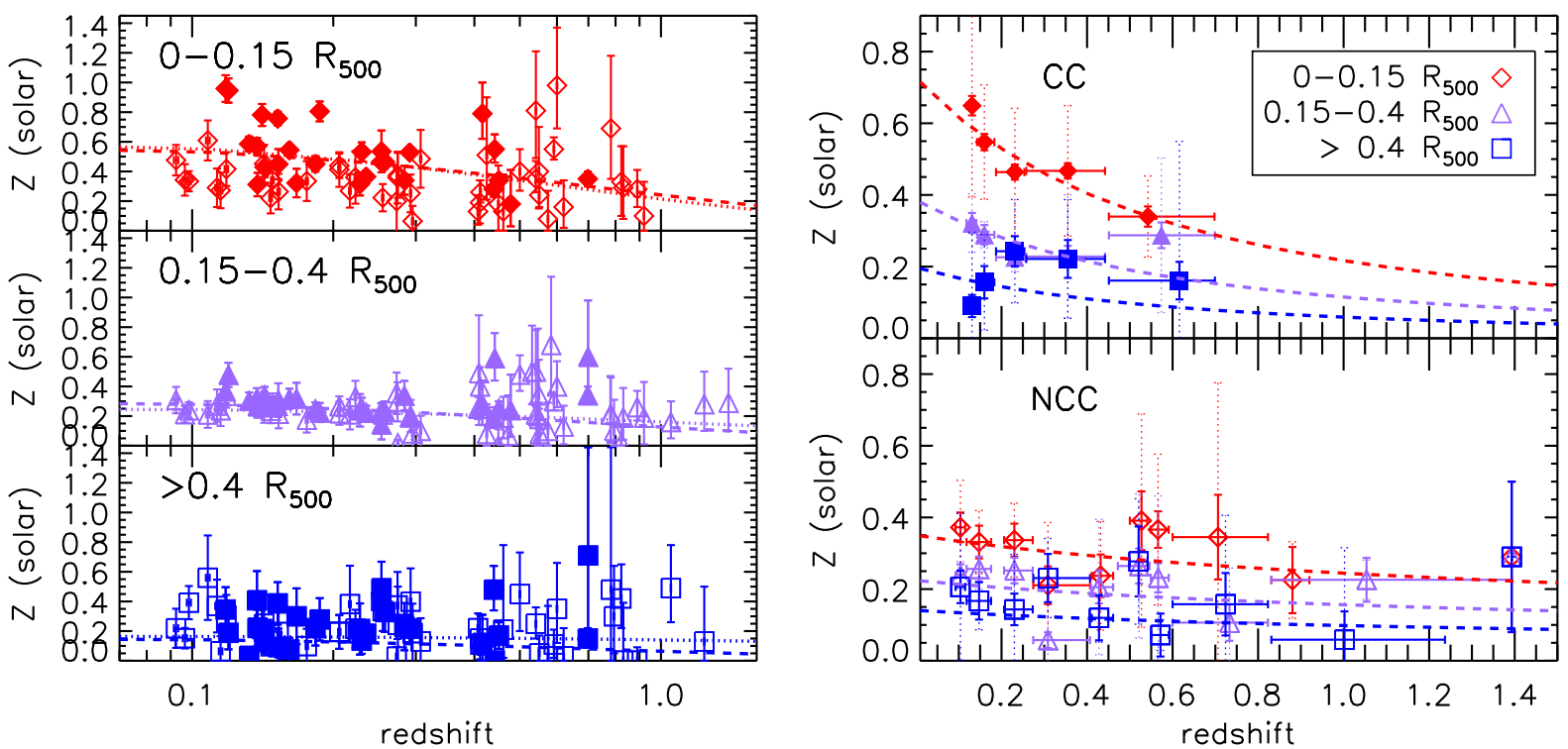

Fig. 1. Abundance distribution as function of redshift as estimated in three radial bins $\left(0-0.15 R_{500}, 0.15-0.4 R_{500}\right.$, and $\left.>0.4 R_{500}\right)$. Filled points indicate CC clusters. Left: all data are plotted with the best fits done in the given radial bin (dotted line) and including the radial dependence (dashed line); right: the best fits in redshift and radius (dashed lines) are shown with representative data points obtained as a weighted mean, with the relative error (solid bars) and dispersion (dashed bars).

Table 1. Results of the combined analysis on the redshift and radial distribution of the metal abundance.

\begin{tabular}{|c|c|c|c|c|c|c|c|}
\hline Sample & $N$ & $\chi^{2}$ & $\sigma$ & $Z_{0}$ & $\gamma$ & $\beta$ & Constant \\
\hline $0-0.15 R_{500}$ & 70 & 267.1 & $0.151 \pm 0.020$ & $0.648 \pm 0.031\left(0.647_{-0.073}^{+0.082}\right)$ & $1.60 \pm 0.22\left(1.59_{-0.43}^{+0.49}\right)$ & - & $0.451 \pm 0.009(0.213)$ \\
\hline Only CC & 27 & 140.7 & $0.159 \pm 0.031$ & $0.795 \pm 0.046\left(0.780_{-0.095}^{+0.098}\right)$ & $2.19 \pm 0.28\left(2.10_{-0.52}^{+0.51}\right)$ & - & $0.493 \pm 0.011(0.207)$ \\
\hline Only NCC & 43 & 50.6 & $0.053 \pm 0.038$ & $0.360 \pm 0.041\left(0.365_{-0.044}^{+0.050}\right)$ & $0.45 \pm 0.40\left(0.51_{-0.59}^{+0.71}\right)$ & - & $0.320 \pm 0.019(0.187)$ \\
\hline $0.15-0.4 R_{500}$ & 83 & 147.7 & $0.044 \pm 0.012$ & $0.261 \pm 0.022\left(0.271_{-0.042}^{+0.035}\right)$ & $0.70 \pm 0.32\left(0.66_{-0.49}^{+0.65}\right)$ & - & $0.220 \pm 0.009(0.134)$ \\
\hline Only CC & 29 & 27.9 & $0.012 \pm 0.025$ & $0.287 \pm 0.032\left(0.293_{-0.039}^{+0.072}\right)$ & $0.30 \pm 0.47\left(0.33_{-0.58}^{+1.26}\right)$ & - & $0.269 \pm 0.013(0.120)$ \\
\hline Only NCC & 54 & 96.8 & $0.048 \pm 0.017$ & $0.197 \pm 0.027\left(0.218_{-0.074}^{+0.053}\right)$ & $0.37 \pm 0.46\left(0.37_{-0.77}^{+0.63}\right)$ & - & $0.178 \pm 0.012(0.146)$ \\
\hline$>0.4 R_{500}$ & 68 & 74.4 & $0.029 \pm 0.028$ & $0.168 \pm 0.028\left(0.173_{-0.044}^{+0.068}\right)$ & $0.26 \pm 0.61\left(0.33_{-0.82}^{+0.88}\right)$ & - & $0.158 \pm 0.014(0.170)$ \\
\hline Only CC & 27 & 40.1 & $0.071 \pm 0.038$ & $0.131 \pm 0.031\left(0.138_{-0.043}^{+0.106}\right)$ & $-0.88 \pm 0.84\left(-0.71_{-1.04}^{+1.41}\right)$ & - & $0.158 \pm 0.019(0.183)$ \\
\hline Only NCC & 41 & 30.7 & $0.000 \pm 0.008$ & $0.226 \pm 0.053\left(0.223_{-0.047}^{+0.056}\right)$ & $1.39 \pm 0.92\left(1.31_{-0.94}^{+0.85}\right)$ & - & $0.157 \pm 0.020(0.164)$ \\
\hline All & 221 & 506.3 & $0.092 \pm 0.010$ & $0.702 \pm 0.031\left(0.699_{-0.079}^{+0.091}\right)$ & $1.33 \pm 0.18\left(1.31_{-0.36}^{+0.41}\right)$ & $0.56 \pm 0.03\left(0.55_{-0.08}^{+0.09}\right)$ & $0.300 \pm 0.006(0.181)$ \\
\hline Only CC & 83 & 225.1 & $0.105 \pm 0.015$ & $0.838 \pm 0.046\left(0.831_{-0.113}^{+0.127}\right)$ & $1.75 \pm 0.24\left(1.72_{-0.53}^{+0.55}\right)$ & $0.55 \pm 0.04\left(0.55_{-0.07}^{+0.07}\right)$ & $0.365 \pm 0.008(0.200)$ \\
\hline Only NCC & 138 & 185.3 & $0.037 \pm 0.013$ & $0.387 \pm 0.038\left(0.393_{-0.042}^{+0.043}\right)$ & $0.52 \pm 0.29\left(0.53_{-0.42}^{+0.45}\right)$ & $0.39 \pm 0.06\left(0.37_{-0.10}^{+0.15}\right)$ & $0.207 \pm 0.009(0.174)$ \\
\hline
\end{tabular}

Notes. The columns show: the selected sample; the number of fitted data points; the total $\chi^{2}$ value; the intrinsic scatter estimated by requiring that the reduced $\chi^{2}$ is equal to 1 ; the best-fit parameters of the adopted functional form $Z(r, z)=Z_{0}\left(1+\left(r / 0.15 R_{500}\right)^{2}\right)^{-\beta}(1+z)^{-\gamma}$. Median, 1 st and 3rd quartiles from the distribution of the best-fitting parameters in the bootstrap analysis over a sample of $10^{5}$ repetitions are indicated in round parentheses. In the last column, labelled "constant", we list the weighted-mean, its relative error and the dispersion around it in round parentheses.

Eighty-three data points are located in the radial bin $0.15 R_{500}<r<0.4 R_{500}$, where a $2.8 \sigma$ significant correlation between pseudo-entropy ratio and redshift is detected, suggesting an increase of $\sigma$ (i.e larger incidence of NCC systems) with the redshift. A mild anti-correlation between $Z$ and redshift is also present in this bin (significance of $2.2 \sigma$ ). We measure $\gamma=0.70 \pm 0.32$ ( $1 \sigma$ range of $0.16-1.31$ after bootstrap analysis). The case for negative evolution is still observed in about $91 \%$ of the bootstrapped samples. Similar values are measured in the population of NCC clusters, which dominates the sample in this radial bin, with a normalization (and a mean metallicity value) that is lower than the corresponding value measured for CC systems by about 30 per cent.

Finally, we do not find evidence of negative redshift evolution of the metallicity in the last radial bin considered in our analysis $\left(r>0.4 R_{500}\right)$, either. In this case, we constrain a value for $\gamma$ of $0.26 \pm 0.61(\gamma>0$ with a probability $P \approx 65 \%$ after bootstrap analysis) with 68 data points. Similar results are obtained in the $\mathrm{CC}$ and NCC populations, where an average value of $Z=0.16 Z_{\odot}$ is estimated.

As the sample is highly heterogeneous and not in any sense representative of the cluster population, it is not expected to 
Table 2. Spearman's rank correlation coefficients.

\begin{tabular}{ccccc}
\hline \hline Sample & $\rho_{z}$ & $\rho_{T}$ & $\rho_{\sigma}$ & $\rho_{\sigma, z}$ \\
\hline $0-0.15 R_{500}$ & $-0.25(2.07)$ & $-0.31(2.54)$ & $-0.39(3.27)$ & $0.18(-1.51)$ \\
$0.15-0.4 R_{500}$ & $-0.24(2.18)$ & $-0.13(1.14)$ & $-0.13(1.18)$ & $0.31(-2.80)$ \\
$>0.4 R_{500}$ & $-0.20(1.63)$ & $-0.09(0.75)$ & $-0.10(0.84)$ & $0.23(-1.85)$ \\
All & $-0.20(3.04)$ & $-0.09(1.27)$ & $-0.20(2.93)$ & $0.25(-3.66)$ \\
\hline
\end{tabular}

Notes. The Spearman's rank correlation coefficients (and the significance in terms of the standard deviations by which the sum-squared difference of ranks deviates from its null-hypothesis expected value) between metallicity and redshift $\left(\rho_{z}\right)$, metallicity and temperature $\left(\rho_{T}\right)$, metallicity and pseudo-entropy ratio $\left(\rho_{\sigma}\right)$, and pseudo-entropy ratio and redshift $\left(\rho_{\sigma, z}\right)$, respectively. The pseudo-entropy ratio is larger in NCC systems.

weigh properly against the relative incidence of these cool cores clusters. Moving at higher redshift, for instance, lower metal abundance associated with the inner regions suggest that less pronounced cool cores are resolved in our sample (see e.g. Santos et al. 2010; Samuele et al. 2011).

\section{Summary and conclusions}

We present the statistical analysis of the spatially resolved spectroscopic results of the $X M M-N e w t o n$ observations of 83 clusters of galaxies in the redshift range $0.09<z<1.39$ and with a gas temperature between $2 \mathrm{keV}$ and $12.8 \mathrm{keV}$. In a consistent and similar way (see details in Leccardi \& Molendi 2008; Baldi et al. 2012a), we resolved the cluster emission for all objects in the data set in three different regions: the core region (corresponding to $0<r<0.15 R_{500}$ ), the region immediately surrounding the core $\left(0.15 R_{500}<r<0.4 R_{500}\right)$, and the outskirts of the cluster $\left(r>0.4 R_{500}\right)$. Using the pseudo-entropy ratio $\sigma$, we also distinguish between cool core and non-cool core objects, with CC clusters that represent $\sim 35$ per cent of the whole sample. Our results are summarized in Tables 1 and 2.

We find strong (significance at $3 \sigma$ or higher) correlations between (i) metallicity and pseudo-entropy ratio (in the inner bin) and, in the entire sample; (ii) metallicity and redshift; and (iii) pseudo-entropy ratio and redshift.

By fitting a constant value (under the assumption of noevolution) to the metal abundance measured in the three radial bins, we confirm a neat decrease moving outwards, with the inner bin being even three (two) times more metal rich than the outermost bin in CC (NCC) systems $(0.49 / 0.32,0.27 / 0.18$ and $0.16 / 0.16$ for $\mathrm{CC} / \mathrm{NCC}$ systems in the three radial bins, respectively).

We constrain the parameters of the functional form $Z(r, z)=$ $Z_{0}\left(1+\left(r / 0.15 R_{500}\right)^{2}\right)^{-\beta}(1+z)^{-\gamma}$. The best-fitting values are $Z_{0}=0.70 \pm 0.03(0.62-0.79,1$ st -3 rd quartile from the bootstrap analysis), $\beta=0.56 \pm 0.03(0.48-0.65)$ and $\gamma=1.3 \pm 0.2$ $(0.95-1.72)$. The CC and NCC systems behave differently, with $\left(Z_{0}, \beta, \gamma\right)$ equal to $(0.83 \pm 0.13,0.55 \pm 0.07,1.7 \pm 0.6)$ and $(0.39 \pm$ $0.04,0.37 \pm 0.15,0.5 \pm 0.5$ ), respectively (error bars refer to the bootstrap analysis).

At a given radius, we find a statistically significant negative evolution in the distribution of the metal abundance as a function of redshift in the inner radial bin $(>3.5 \sigma$; the probability from the bootstrap analysis to measure $\gamma>0$ is larger than 99.9 per cent). We show that this level of evolution with cosmic time is entirely consistent with the same evolution observed in the CC-only population. On average, we measure a mean metallicity in the local systems that is about 3-4.5 (1.4 in NCC clusters) times the value measured at $z \approx 1$, which is consistent with the constraints we publish in Baldi et al. (2012a) and, using (mostly) Chandra data, in Balestra et al. (2007). At the same redshift, the metallicity decreases radially, reaching at $\sim 0.5 R_{500}$ about 37 (in CC) and 50 (in NCC) per cent of the value estimated at $0.15 R_{500}$. By requiring a reduced $\chi^{2}$ of 1 in the performed fit, we estimate an intrinsic scatter in $Z$ of about $0.15,0.05$ and 0.05 in the three radial bins, respectively. In the inner bin, the scatter of $\sim 0.15$ is also measured only in the CC systems, whereas NCC clusters show a value of 0.05 consistently in the inner two radial bins.

The CC clusters show evidence of negative evolution just in the inner radial bin $\left(<0.15 R_{500}\right)$, where the accumulation of the plasma during the cluster formation makes the ICM denser and, as consequence of radiative processes, cooler and more metal rich. Most of the above-mentioned correlations (i.e. $\rho_{z}, \rho_{T}$ and $\rho_{\sigma}$ in Table 2) can be then interpreted as due to the strong influence of this centrally-located cool gas. The NCC systems do not show any statistically significant deviation from $\gamma=0$ at any radial bin. Both CC and NCC systems present similar average metallicity, and an intrinsic scatter almost consistent with zero, in the outer bin $\left(r>0.4 R_{500}\right)$ only. We conclude that, for what concerns the metal distribution, this cluster volume is the most homogeneous in both CC and NCC systems, and might be considered the most representative of the average cluster environment.

We stress that this work provides the most robust constraints reachable with archived XMM-Newton and Chandra data on the distribution of the metal abundance as a function of radius and redshift in the ICM. Larger samples of high redshift X-ray clusters, together with deeper Chandra and XMM-Newton observations even of the clusters already present in the archive, would be crucial to putting tighter constraints on the evolution of the ICM metal abundance. These samples are also needed to provide a robust modelling of the physical processes responsible for the enrichment of the cluster plasma during its assembly over cosmic time, from the accumulation of the iron mass in the $\mathrm{CC}$ cluster cores (e.g. De Grandi et al. 2004; Böhringer et al. 2004) to the history of the processes responsible for the metal release into the ICM through ejections from supernovae Types Ia and II (e.g. Ettori 2005), as well as through galaxy transformation in the cluster volume (e.g. Calura et al. 2007) and their modelling in joint semi-analytic/hydrodynamical numerical simulations (e.g. Cora et al. 2008; Fabjan et al. 2010).

Acknowledgements. We thank Sabrina De Grandi and Mauro Sereno for their discussion. We acknowledge financial contribution from contracts ASI-INAF I/009/10/0 and Prin-INAF 2012 on "A unique data set to address the most compelling open questions about X-Ray Galaxy Clusters". We thank the anonymous referee for useful comments that helped to improve the presentation of the results in this paper.

\section{References}

Anders, E., \& Grevesse, N. 1989, Geochim. Cosmochim. Acta, 53, 197 Anderson, M. E., Bregman, J. N., Butler, S. C., \& Mullis, C. R. 2009, ApJ, 698,317

Andreon, S. 2012, A\&A, 546, A6

Arnaud, K. A. 1996, in Astronomical Data Analysis Software and Systems V, eds. G. H. Jacoby, \& J. Barnes, ASP Conf. Ser., 101, 17

Baldi, A., Ettori, S., Mazzotta, P., Tozzi, P., \& Borgani, S. 2007, ApJ, 666, 835 Baldi, A., Ettori, S., Molendi, S., et al. 2012a, A\&A, 537, A142

Baldi, A., Ettori, S., Molendi, S., \& Gastaldello, F. 2012b, A\&A, 545, A41 Balestra, I., Tozzi, P., Ettori, S., et al. 2007, A\&A, 462, 429

Böhringer, H., Matsushita, K., Churazov, E., Finoguenov, A., \& Ikebe, Y. 2004, A\&A, 416, L21 
S. Ettori et al.: The evolution of the metal abundance up to $z=1.4(R N)$

Calura, F., Matteucci, F. \& Tozzi, P. 2007, MNRAS, 378, L11

Cora, S. A., Tornatore, L., Tozzi, P., \& Dolag, K. 2008, MNRAS, 386, 96

De Grandi, S., \& Molendi, S. 2001, ApJ, 551, 153

De Grandi, S., Ettori, S., Longhetti, M., \& Molendi, S. 2004, A\&A, 419, 7

Ettori, S. 2005, MNRAS, 362, 110

Fabjan, D., Borgani, S., Tornatore, L., et al. 2010, MNRAS, 401, 1670

Finoguenov, A., David, L. P., \& Ponman, T. J. 2000, ApJ, 544, 188

Irwin, J. A., \& Bregman, J. N. 2001, ApJ, 546, 150

Kalberla, P. M. W., Burton, W. B., Hartmann, D., et al. 2005, A\&A, 440, 775

Leccardi, A., \& Molendi, S. 2008, A\&A, 487, 461

Leccardi, A., Rossetti, M., \& Molendi, S. 2010, A\&A, 510, A82
Maughan, B. J., Jones, C., Forman, W., \& Van Speybroeck, L. 2008, ApJS, 174, 117

Rasia, E., Mazzotta, P., Bourdin, H., et al. 2008, ApJ, 674, 728

Renzini, A. 1997, ApJ, 488, 35

Samuele, R., McNamara, B. R., Vikhlinin, A., \& Mullis, C. R. 2011, ApJ, 731, 31

Santos, J. S., Tozzi, P., Rosati, P., \& Böhringer, H. 2010, A\&A, 521, A64

Schindler, S., \& Diaferio, A. 2008, Space Sci. Rev., 134, 363

Snowden, S. L., Mushotzky, R. F., Kuntz, K. D., \& Davis, D. S. 2008, A\&A, 478,615

Tamura, T., Kaastra, J. S., den Herder, J. W. A., Bleeker, J. A. M., \& Peterson, J. R. 2004, A\&A, 420, 135

Vikhlinin, A., Kravtsov, A., Forman, W., et al. 2006, ApJ, 640, 691 